\title{
Consumption of sugar-sweetened beverages and its association with overweight among young children from China
}

\author{
Pan Yu ${ }^{1}$, Yun $\mathrm{Chen}^{2}, \mathrm{Ai}$ Zhao ${ }^{1}$, Ying Bai ${ }^{2, *}$, Yingdong Zheng ${ }^{1}$, Wenzhi Zhao ${ }^{1}$ and \\ Yumei Zhang ${ }^{1, *}$ \\ 'Department of Nutrition and Food Hygiene, School of Public Health, Peking University Health Science Center, Room \\ 415, 38 Xueyuan Road, Haidian District, Beijing 100191, People's Republic of China: ${ }^{2}$ Dairy Research Institute, \\ Inner Mongolia Mengniu Dairy (Group) Co. Ltd, Helingeer Shengle Economic District, Hohhot 011500 , Inner \\ Mongolia, People's Republic of China
}

Submitted 9 0ctober 2015: Final revision received 1 March 2016: Accepted 7 April 2016: First published online 6 June 2016

\begin{abstract}
Objective: To fully understand the sugar-sweetened beverage (SSB) consumption status among Chinese young children and to explore its association with weight gain.

Design: In this cross-sectional study, data on sociodemographic characteristics, SSB intake and weight/height were collected by means of face-to-face interviews. The intake of SSB among young Chinese children in relation to their age, different characteristics and types of SSB consumed is described, and the association between SSB intake and BMI-for-age $Z$-score and overweight is explored.

Setting: Seven large cities and two villages in China.

Subjects: Nine hundred and forty-six healthy children, aged 3-7 years.

Results: The proportion of SSB intake among Chinese young children was $80.5 \%$; $3.4 \%$ were daily consumers, $34.0 \%(31.4 \%)$ consumed at least once per week (month). The per capita and per consumer SSB intake was 63.1 9 (SD 100.8) and 78.4 (SD 106.9) $\mathrm{ml} / \mathrm{d}$. Children from rural areas consumed twice, or even triple, the amount of SSB as those from urban areas $(P<0.001)$ and great disparities existed between the types of SSB consumed by urban and rural children. An association was found between increased SSB intake and higher BMI-for-age $Z$-score $(P<0.05)$ after adjusting for potential confounders; there was also an association between SSB intake and increased risk of being overweight or obese.

Conclusions: The consumption status of SSB in Chinese young children is of concern. There was a positive association between SSB intake and weight gain. Measures should be taken to improve the present situation of SSB consumption among Chinese young children.
\end{abstract}

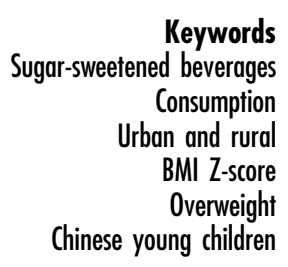

There has been a dramatic rise in the number of children who are overweight. According to the 2013 UNICEF, WHO and World Bank estimates, between 2000 and 2013 the number of overweight children worldwide increased from 32 million to 42 million. The prevalence of childhood overweight is increasing in all regions, particularly in Africa and Asia. Between 2000 and 2013, the prevalence of overweight in children under 5 years of age increased from 3 to $7 \%$ in South-East Asia $^{(1)}$. This reflects the increasing trend in China; from 1981 to 2010, the prevalence of overweight and obesity among children increased from 1.8 (95\% CI $0.4,3.1) \%$ and 0.4 (95\% CI -0.1, -0.8) \% respectively in 1981-1985, to 13.1 (95\% CI $11 \cdot 2,15 \cdot 0$ ) \% and 7.5 (95\% CI 6.6, 8.4) \% respectively in 2006-2010. The average annual increase was 8.3 and $12.4 \%$, respectively ${ }^{(2)}$.
Overweight and obesity in the paediatric population has attracted much attention because of its persistence into adulthood $^{(3)}$ and short- and long-term adverse health consequences $^{(4)}$. The reasons for overweight and obesity are complex, comprising the interplay of genetic, behavioural and environmental influences on metabolism, diet and activity. Nowadays, multiple sources have noted that the trends towards increasing overweight and obesity rates worldwide have paralleled an increase in sugar intake from food ${ }^{(5,6)}$. One common source of excess sugar consumption is sugar-sweetened beverages (SSB), such as carbonated drinks, fruit and/or vegetable drinks and tea. The relationship between SSB intake and overweight and obesity has been widely studied; however, the evidence is still insufficient to produce a definitive conclusion. 
For example, in their systematic review on the association between SSB consumption and risk of obesity, Trumbo and Rivers found that the results (adjusted for energy intake and physical activity) were inconsistent for the evaluated groups of children, adolescents and adults ${ }^{(7)}$. Similar results were obtained from other studies and reviews ${ }^{(8,9)}$. Furthermore, Forshee et al. in their quantitative meta-analysis and qualitative review, based on the current body of scientific evidence, found the association between SSB consumption and BMI was near zero ${ }^{(10)}$. However, several other studies and reviews reported a positive relationship between SSB intake and weight gain $^{(11,12)}$; for example, Ludwig et al. concluded that, for each additional serving of SSB consumed, both BMI and frequency of obesity (OR $=1.60 ; 95 \%$ CI 1.14, 2.24; $P=0.02)$ increased after adjustment for anthropometric, dietary, demographic and lifestyle factors ${ }^{(13)}$. Similarly, DeBoer et al. found that higher rates of SSB consumption were associated with higher BMI $Z$-scores among children aged 4 years $(P<0.05)$ and 5 years $(P<0 \cdot 001)$. Children aged 5 years who drank SSB regularly had a higher odds for being obese compared with infrequent or non-drinkers $(\mathrm{OR}=1.43 ; 95 \% \text { CI } 1.10,1.85 ; P<0 \cdot 01)^{(14)}$.

In China, studies on the relationship between SSB intake and overweight/obesity are limited and most of them have focused on adults; little is known about the SSB intake among young children ${ }^{(15-17)}$. Furthermore, with the development of urbanisation, increasing numbers of children in rural areas are becoming overweight or obese. From 1991 to 2000, urbanisation was strongly and positively associated with increased BMI, but from 2000 to 2011 the overweight/obesity trends became more similar across rural and urban areas ${ }^{(18)}$. The data are limited on whether there is a difference between urban and rural children regarding SSB intake.

We performed the present study to: (i) establish the consumption status of SSB among young children in urban and rural areas in China, and the differences between them; and (ii) investigate the relationship between SSB intake and overweight/obesity among young Chinese children.

\section{Materials and methods}

\section{Study sample}

Data for the present study were collected between November 2011 and April 2012 from healthy pre-schoolers in China, and included a total sample of 1055 children aged 3-7 years. A multistage, stratified, cluster sampling method was used. First, considering specific geographic location and economic status, seven cities were selected, including Beijing, Suzhou, Guangzhou, Zhengzhou, Chengdu, Shenyang and Lanzhou, and two villages including one in a flat and another in a mountainous area, both located in Xingtai, Hebei Province. Second, one large kindergarten located in each city or village was selected randomly. Third, in each selected kindergarten, one bottom, one middle and one top class were selected randomly; all individuals within the classes were selected for the survey.

The inclusion criteria were healthy children aged 3-7 years old; the exclusion criteria were: (i) reported birth defects including congenital heart disease, hydrocephalus or deformity at birth; (ii) reported infantile paralysis and thalassaemia; and (ii) acute health problems including cold and diarrhoea at the time of sampling.

All 1055 healthy pre-schoolers participated in our survey. Ninety-one participants did not finish the beverage intake questionnaire, twelve did not provide information on sex, four did not provide their height and weight data, and two had a BMI above $50 \mathrm{~kg} / \mathrm{m}^{2}$. These preschoolers were excluded. Consequently, a final total of 946 participants were included in the present research.

\section{Sociodemographic characteristics, dietary babits and antbropometry}

After determining eligibility, a research assistant contacted families and arranged a meeting with parents for a faceto-face interview. Sociodemographic information was collected from the parents by means of a structured questionnaire, administered by trained interviewers. Demographic data included the child's date of birth, sex, ethnicity and parents' educational level - (i) illiteracy; (ii) primary school; (iii) middle school; (iv) senior high school; (v) college; (vi) graduate or above - as well as family income (per capita monthly income) - (i) below 2000 Yuan; (ii) 2000-4000 Yuan; (iii) 4000-8000 Yuan; (iv) above 8000 Yuan. Parents' body weights and heights were self-reported from the interview.

Dietary habits affect the selection of foods and hence may influence children's growth and development. In the present research, picky eating behaviour and meals outside the home particularly attracted our attention. We defined a picky eater as a child who consumed an inadequate variety and amount of food(s) through rejection of foods that were familiar (and unfamiliar) to them. The definition was provided to parents before the picky eating assessment and parents' perceptions were used to judge if their child was a picky eater. They were asked 'Do you consider your child as having picky eating behaviour at present?' Possible answers were 'yes' and 'no'. In the meals outside the home section, parents were asked 'On average, how many times do you take your child to eat outside every week?' Possible answers were ' 0 ', ' 1 time', ' 2 times' and ' 3 or more times'.

All anthropometric measurements were performed by trained investigators following standardised procedures $^{(19)}$. The child's weight with light clothes was measured to the nearest $0.1 \mathrm{~kg}$ on a calibrated electronic scale (EKS 7028; Beaver International Holding Ltd, Hong Kong, China) and standing height was taken to the nearest $0.1 \mathrm{~cm}$ 
using a calibrated height measuring tape (SECA 206; SECA Ltd, Hamburg, Germany) suspended from the wall. All physical measurements were measured three times continuously and the mean values were used.

\section{Beverage consumption}

A beverage intake frequency questionnaire was provided to obtain information on the children's consumption of beverages. Each beverage was categorised based on nutrient content and on China's Beverage General Rule (GB10789-2008). Fourteen broad beverage categories were used: (i) whole milk; (ii) skimmed/low-fat milk; (iii) yoghurt; (iv) lactobacillus beverage; (v) milk beverage (such as Nutrition Express); (vi) plant protein beverage; (vii) carbonated beverage; (viii) 100\% fruit or vegetable juice; (ix) fruit and/or vegetable beverage; $(\mathrm{x}$ ) packaged drinking water; (xi) tea; (xii) tea beverage; (xiii) coffee beverage; and (xiv) sports/energy drinks (such as Mizone). Parents were asked by trained investigators how often (never, every half year, every month, every week or every day), how many times and how much amount, of the specific groups of beverage their child had consumed during the preceding half year.

We investigated only the non-diary sugar-sweetened beverages in the present study.

\section{Definitions}

Since there is no unified definition of SSB, in the present research we took into account the classification of SSB presented in other documents ${ }^{(14,20,21)}$ and consumption habits in China. An SSB was clarified as being a carbonated beverage (e.g. Coke, Pepsi or Sprite); a vegetable- or fruit-flavoured beverage that was not $100 \%$ fruit or vegetable juice; a tea beverage (e.g. iced tea, Jiaduobao); and sports/energy drinks.

The children's BMI was calculated as mass in kilograms divided by the square of height in metres $\left(\mathrm{kg} / \mathrm{m}^{2}\right) . Z$-scores for BMI-for-age of the children were obtained using the software WHO Anthro ${ }^{(22,23)}$. Overweight and obesity was defined using WHO standards. For children under 5 years, a BMI-for-age $Z$-score above +2 but below or equal to +3 was classified as overweight. Obesity was defined as BMI-for-age $Z$-score above $+3^{(24)}$. For children aged above 5 years, overweight was classified as BMI-for-age $Z$-score above +1 but below or equal to +2 . Obesity was defined as BMI-forage $Z$-score above $+2^{(25)}$. Regarding the parents, overweight was defined as BMI greater than or equal to $25 \cdot 0 \mathrm{~kg} / \mathrm{m}^{2}$.

\section{Statistical analysis}

All statistical analyses were performed using the statistical software package IBM SPSS Statistics Version 19. Continuous variables were presented as mean and standard deviation. Differences in the proportions of SSB consumption between groups by subject characteristics were analysed by the $\chi^{2}$ test, and the $t$ test or one-way ANOVA (Mann-Whitney $U$ test or Kruskal-Wallis $H$ test for nonnormally distributed data) was used to test differences in the amount of SSB intake between groups by subject characteristics. Using multivariable linear regression models, we explored the association of different levels of SSB intake and BMI $Z$-score, and logistic regression models were used to examine the odds of SSB consumers being overweight or obese. A $P$ value of $<0.05$ was considered significant.

\section{Results}

A total of 946 young children from seven cities and two villages in China were included. The average age of the children was 4.84 (SD 0.89) years; 190 were aged 3-3.9 years, 337 were aged 4-4.9 years, 299 were aged 5-5.9 years and 120 were aged 6-7 years; $493(52 \cdot 1 \%)$ were boys; and $721(76 \cdot 2 \%)$ were from urban areas.

\section{Social factors and sugar-sweetened beverage consumption}

Tables 1, 2 and 3 show the consumption of SSB among young Chinese children, by age, according to different characteristics. The total percentage of SSB consumption was $80.5 \%$ during the half year preceding the study. Among all consumers, $3.4 \%$ consumed SSB at least once daily, $34.0 \%$ at least once weekly, $31.4 \%$ at least once monthly and $11.8 \%$ at least once biannually. Per capita and per consumer SSB intake was $63 \cdot 1$ (SD 100.8) and 78.4 (sD 106.9$) \mathrm{ml} / \mathrm{d}$, respectively. There were no significant differences between the different age groups regarding the percentage of SSB consumption, per capita and per consumer SSB daily intake $(P=0.688,0.209$ and 0.239, respectively).

Among all the studied children, sex, child's area, picky eating behaviour, parents' education level and family income were important factors associated with children's SSB consumption status. There was no significant difference in the percentage of boys and girls consuming SSB. However, compared with girls, the amount of SSB consumed by boys was much larger. Concerning child's area, it was clearly seen that children from rural areas consumed nearly twice the amount of SSB as those from urban areas. Children with a picky eating habit consumed more SSB than those who were not picky. Parents' education level was relevant; children consumed less SSB if their parents' education level was higher.

In different age groups, children's area and parents' education level were the most important factors that influenced children's SSB consumption. Among all the age groups, children from rural areas consumed nearly twice - and even triple, in the case of 3-year-old children - the amount of SSB as those from urban areas. 
Table 1 Total consumption of sugar-sweetened beverages, according to different characteristics, by young children ( $n$ 946) aged 3-7 years from seven large cities and two villages in China, November 2011-April 2012

\begin{tabular}{|c|c|c|c|c|c|c|}
\hline \multirow[b]{2}{*}{ Characteristic } & \multirow[b]{2}{*}{ Sample size } & \multirow[b]{2}{*}{ Consumer (\%) } & \multicolumn{2}{|c|}{ Per capita (ml/d) } & \multicolumn{2}{|c|}{ Per consumer $(\mathrm{ml} / \mathrm{d})$} \\
\hline & & & Mean & SD & Mean & SD \\
\hline Total & 946 & $80 \cdot 5$ & $63 \cdot 1$ & $100 \cdot 8$ & $78 \cdot 4$ & $106 \cdot 9$ \\
\hline \multicolumn{7}{|l|}{ Child's sex } \\
\hline Male & 493 & $81 \cdot 1$ & $72 \cdot 6^{*}$ & 118.5 & $89 \cdot 5^{*}$ & $125 \cdot 7$ \\
\hline Female & 453 & 79.9 & $52 \cdot 8$ & $75 \cdot 9$ & $66 \cdot 1$ & $79 \cdot 6$ \\
\hline \multicolumn{7}{|l|}{ Child's area } \\
\hline Urban & 721 & 79.5 & $50 \cdot 2^{*}$ & $87 \cdot 2$ & $63 \cdot 2^{*}$ & 93.6 \\
\hline Rural & 225 & $84 \cdot 0$ & 104.5 & $127 \cdot 2$ & 124.5 & $129 \cdot 6$ \\
\hline \multicolumn{7}{|l|}{ Picky eating behaviour } \\
\hline Yes & 496 & $84 \cdot 1^{*}$ & $75 \cdot 0^{*}$ & $118 \cdot 0$ & $89 \cdot 2^{*}$ & 123.7 \\
\hline No & 435 & $76 \cdot 8$ & $50 \cdot 6$ & 76.4 & 65.9 & $81 \cdot 2$ \\
\hline \multicolumn{7}{|l|}{ Mother's education } \\
\hline Primary school or below & 49 & $73 \cdot 5^{\star}$ & $61 \cdot 7^{\star}$ & $89 \cdot 7$ & $84 \cdot 0^{\star}$ & 95.5 \\
\hline Middle-school diploma & 266 & $84 \cdot 2$ & $94 \cdot 6$ & 133.4 & $112 \cdot 3$ & $138 \cdot 4$ \\
\hline High-school diploma & 423 & $82 \cdot 5$ & 55.7 & 86.9 & 67.5 & 91.4 \\
\hline College degree and above diploma & 187 & $72 \cdot 2$ & $34 \cdot 2$ & $62 \cdot 1$ & $47 \cdot 4$ & $68 \cdot 7$ \\
\hline \multicolumn{7}{|l|}{ Mother's BMI $\left(\mathrm{kg} / \mathrm{m}^{2}\right)$} \\
\hline$<18.5$ & 106 & $77 \cdot 4$ & $54 \cdot 0$ & $118 \cdot 1$ & $69 \cdot 8$ & $130 \cdot 3$ \\
\hline $18 \cdot 5-24.9$ & 719 & 81.4 & 63.0 & 97.8 & 77.5 & $103 \cdot 1$ \\
\hline$\geq 25 \cdot 0$ & 93 & $80 \cdot 6$ & $79 \cdot 8$ & $111 \cdot 0$ & $99 \cdot 0$ & $115 \cdot 7$ \\
\hline \multicolumn{7}{|l|}{ Father's education } \\
\hline Primary school or below & 26 & $65 \cdot 4^{*}$ & $110 \cdot 2^{*}$ & 211.9 & $168 \cdot 6^{\star}$ & 244.3 \\
\hline Middle-school diploma & 241 & 85.9 & $101 \cdot 1$ & $127 \cdot 0$ & $117 \cdot 8$ & $129 \cdot 7$ \\
\hline High-school diploma & 408 & $82 \cdot 6$ & 54.8 & 81.2 & 66.4 & 85.0 \\
\hline College degree and above diploma & 227 & $73 \cdot 1$ & $32 \cdot 0$ & 63.6 & 43.8 & $70 \cdot 9$ \\
\hline \multicolumn{7}{|l|}{ Father's BMI $\left(\mathrm{kg} / \mathrm{m}^{2}\right)$} \\
\hline$<18.5$ & 18 & $72 \cdot 2$ & $42 \cdot 6^{*}$ & $59 \cdot 2$ & $59 \cdot 0$ & $62 \cdot 7$ \\
\hline $18 \cdot 5-24 \cdot 9$ & 575 & 79.7 & $58 \cdot 2$ & $92 \cdot 3$ & $73 \cdot 1$ & 98.0 \\
\hline$\geq 25 \cdot 0$ & 305 & $82 \cdot 0$ & $75 \cdot 7$ & $118 \cdot 2$ & $92 \cdot 4$ & $124 \cdot 6$ \\
\hline \multicolumn{7}{|l|}{ Family income (Yuan/person per month) } \\
\hline Less than 2000 & 346 & $81 \cdot 8$ & $82 \cdot 7^{*}$ & 124.5 & $101 \cdot 1^{*}$ & $130 \cdot 7$ \\
\hline 2000-4000 & 256 & $75 \cdot 0$ & $45 \cdot 4$ & $75 \cdot 6$ & 60.5 & 81.9 \\
\hline $4000-8000$ & 140 & $82 \cdot 9$ & $51 \cdot 7$ & 81.9 & $62 \cdot 4$ & $86 \cdot 2$ \\
\hline More than 8000 & 39 & $74 \cdot 4$ & $50 \cdot 5$ & $129 \cdot 8$ & $67 \cdot 9$ & $147 \cdot 1$ \\
\hline \multicolumn{7}{|l|}{ Meals outside the home/week } \\
\hline 0 & 244 & $76 \cdot 6$ & 52.5 & $69 \cdot 4$ & $68 \cdot 5$ & $72 \cdot 1$ \\
\hline 1 time & 368 & $82 \cdot 9$ & $62 \cdot 0$ & $103 \cdot 4$ & 74.8 & $109 \cdot 3$ \\
\hline 2 times & 186 & $82 \cdot 3$ & $76 \cdot 9$ & $114 \cdot 2$ & 93.5 & $119 \cdot 6$ \\
\hline 3 or more times & 92 & $80 \cdot 9$ & 63.7 & $97 \cdot 7$ & $78 \cdot 2$ & $102 \cdot 9$ \\
\hline
\end{tabular}

${ }^{*} P<0.05$.

The higher the parents' education level, the less SSB children consumed.

\section{Types of sugar-sweetened beverages consumed}

Among all SSB consumers, carbonated beverages accounted for $22.3 \%$ of all four kinds of SSB; the proportions for tea beverages, vegetable/fruit-flavoured beverages and sports/energy drinks were $42 \cdot 1,13 \cdot 8$ and $21.9 \%$, respectively. Figures 1, 2 and 3 show the types of SSB consumed by children according to area, age and sex. Tea beverages were popular among children from rural areas, where they accounted for more than half $(62.0 \%)$ of all four kinds of SSB, while the corresponding percentage among children from urban areas was $29.1 \%$. Vegetable/fruit-flavoured beverages were much more popular among urban children, accounting for almost a third of all SSB, while the corresponding percentage among rural children was less than $10 \%$
Among different age groups, there was a trend to consume more energy and carbonated beverages, but less tea, with increasing age. The types of SSB consumed by boys and girls were very similar. The most consumed were tea beverages, followed by carbonated beverages, vegetable/ fruit-flavoured beverages and sports/energy drinks.

\section{Sugar-sweetened beverage intake and BMI Z-score/ overweight and obesity}

The average BMI-for-age $Z$-score of the total study population was $0 \cdot 18$ (SD $1 \cdot 64$ ), with children from urban areas having a much higher $Z$-score than those from rural areas (0.34 (sD 1.70) v. -0.34 (sD 1.30); $P<0.001)$. The total prevalence of overweight and obesity was $11.3 \%$; the corresponding morbidity was 13.9 and $3.1 \%$ among urban and rural areas, respectively $(P<0 \cdot 001)$.

Using linear regression, an increasing quantity of SSB consumption was found to be associated with higher BMIfor-age $Z$-score, after adjustment for multiple potential 
Table 2 Consumption of sugar-sweetened beverages, according to different characteristics and age group, by young children ( $n$ 527) from seven large cities and two villages in China, November 2011-April 2012

\begin{tabular}{|c|c|c|c|c|c|c|c|c|c|c|c|c|}
\hline \multirow[b]{3}{*}{ Characteristic } & \multicolumn{6}{|c|}{$3-3.9$ years } & \multicolumn{6}{|c|}{$4-4.9$ years } \\
\hline & \multirow[b]{2}{*}{ Sample size } & \multirow[b]{2}{*}{ Consumer (\%) } & \multicolumn{2}{|c|}{ Per capita $(\mathrm{ml} / \mathrm{d})$} & \multicolumn{2}{|c|}{ Per consumer $(\mathrm{ml} / \mathrm{d})$} & \multirow[b]{2}{*}{ Sample size } & \multirow[b]{2}{*}{ Consumer (\%) } & \multicolumn{2}{|c|}{ Per capita $(\mathrm{ml} / \mathrm{d})$} & \multicolumn{2}{|c|}{ Per consumer $(\mathrm{ml} / \mathrm{d})$} \\
\hline & & & Mean & SD & Mean & SD & & & Mean & SD & Mean & SD \\
\hline Total & 190 & $82 \cdot 1$ & $57 \cdot 1$ & $95 \cdot 7$ & 69.5 & 101.5 & 337 & $78 \cdot 6$ & $56 \cdot 9$ & $91 \cdot 1$ & $72 \cdot 3$ & $97 \cdot 2$ \\
\hline \multicolumn{13}{|l|}{ Child's sex } \\
\hline Male & 95 & $81 \cdot 1$ & $75 \cdot 4^{\star}$ & $119 \cdot 6$ & 93.0* & $126 \cdot 6$ & 190 & 78.9 & $64 \cdot 2$ & $105 \cdot 9$ & $81 \cdot 3$ & $113 \cdot 3$ \\
\hline Female & 95 & $83 \cdot 2$ & 38.8 & $58 \cdot 6$ & $46 \cdot 7$ & 61.4 & 147 & $78 \cdot 2$ & $47 \cdot 3$ & 66.5 & $60 \cdot 5$ & $69 \cdot 7$ \\
\hline \multicolumn{13}{|l|}{ Child's area } \\
\hline Urban & 149 & $78 \cdot 5^{\star}$ & $38.9^{*}$ & 73.7 & $49 \cdot 6^{*}$ & $80 \cdot 0$ & 271 & 77.5 & $49 \cdot 4^{\star}$ & $77 \cdot 4$ & $63 \cdot 8^{*}$ & 82.5 \\
\hline Rural & 41 & $95 \cdot 1$ & 123.2 & $132 \cdot 2$ & 129.5 & $132 \cdot 5$ & 66 & $83 \cdot 3$ & 87.5 & 129.9 & $104 \cdot 9$ & $135 \cdot 8$ \\
\hline \multicolumn{13}{|l|}{ Picky eating behaviour } \\
\hline Yes & 107 & 78.5 & $65 \cdot 0$ & $113 \cdot 7$ & $82 \cdot 9$ & $122 \cdot 6$ & 194 & $85 \cdot 6^{*}$ & $66 \cdot 5^{\star}$ & $100 \cdot 5$ & $77 \cdot 7$ & $104 \cdot 6$ \\
\hline No & 81 & $87 \cdot 7$ & $47 \cdot 3$ & $65 \cdot 3$ & 54.0 & $67 \cdot 2$ & 137 & $70 \cdot 1$ & 44.9 & $76 \cdot 2$ & $64 \cdot 1$ & $84 \cdot 1$ \\
\hline \multicolumn{13}{|l|}{ Mother's education } \\
\hline Primary school or below & 10 & $80 \cdot 0 *$ & $87.9^{\star}$ & $125 \cdot 9$ & $109 \cdot 8^{\star}$ & $132 \cdot 7$ & 21 & $57 \cdot 1^{*}$ & $39 \cdot 2$ & 68.5 & $68 \cdot 6$ & $79 \cdot 6$ \\
\hline Middle-school diploma & 45 & $91 \cdot 1$ & 88.5 & 114.4 & $97 \cdot 1$ & $116 \cdot 4$ & 85 & $82 \cdot 4$ & $76 \cdot 2$ & $121 \cdot 0$ & 92.5 & $127 \cdot 6$ \\
\hline High-school diploma & 82 & 85.4 & 55.5 & $95 \cdot 7$ & $65 \cdot 0$ & $100 \cdot 6$ & 157 & $80 \cdot 9$ & $54 \cdot 0$ & $82 \cdot 4$ & $66 \cdot 7$ & $86 \cdot 9$ \\
\hline College degree and above diploma & 47 & $68 \cdot 1$ & $22 \cdot 0$ & $54 \cdot 0$ & $32 \cdot 2$ & $63 \cdot 1$ & 66 & $72 \cdot 7$ & $44 \cdot 2$ & $70 \cdot 1$ & $60 \cdot 7$ & $76 \cdot 0$ \\
\hline \multicolumn{13}{|l|}{ Mother's BMI $\left(\mathrm{kg} / \mathrm{m}^{2}\right)$} \\
\hline$<18.5$ & 28 & $82 \cdot 1$ & 35.5 & $67 \cdot 1$ & $43 \cdot 3$ & 71.9 & 36 & $72 \cdot 2$ & $40 \cdot 3$ & $80 \cdot 6$ & 55.8 & $90 \cdot 6$ \\
\hline $18.5-24.9$ & 139 & $82 \cdot 0$ & $60 \cdot 0$ & 102.5 & $73 \cdot 1$ & $109 \cdot 0$ & 259 & $80 \cdot 3$ & 57.5 & 90.4 & 71.5 & $95 \cdot 7$ \\
\hline$\geq 25 \cdot 0$ & 19 & 89.5 & $66 \cdot 8$ & $80 \cdot 4$ & 74.7 & 81.6 & 31 & $74 \cdot 2$ & $77 \cdot 2$ & 113.8 & $104 \cdot 0$ & 121.4 \\
\hline \multicolumn{13}{|l|}{ Father's education } \\
\hline Primary school or below & 4 & $75 \cdot 0$ & 73.0* & 88.8 & $97 \cdot 3^{*}$ & $91 \cdot 0$ & 12 & $50 \cdot 0^{*}$ & $44.5^{\star}$ & 74.6 & $89 \cdot 0^{*}$ & 86.5 \\
\hline Middle-school diploma & 42 & 90.5 & $96 \cdot 4$ & $122 \cdot 9$ & $106 \cdot 6$ & $125 \cdot 0$ & 86 & 84.9 & 87.5 & 124.9 & $103 \cdot 1$ & $129 \cdot 5$ \\
\hline High-school diploma & 78 & $82 \cdot 1$ & 44.4 & 61.5 & $54 \cdot 1$ & 63.9 & 153 & $79 \cdot 7$ & 56.5 & $82 \cdot 2$ & $70 \cdot 9$ & 86.4 \\
\hline College degree and above diploma & 52 & $73 \cdot 1$ & $33 \cdot 3$ & $95 \cdot 2$ & $45 \cdot 5$ & $109 \cdot 2$ & 76 & 73.7 & $29 \cdot 9$ & $53 \cdot 3$ & $40 \cdot 5$ & $58 \cdot 6$ \\
\hline \multicolumn{13}{|l|}{ Father's BMI $\left(\mathrm{kg} / \mathrm{m}^{2}\right)$} \\
\hline$<18.5$ & 6 & $50 \cdot 0^{*}$ & $17 \cdot 3$ & $23 \cdot 5$ & $34 \cdot 6$ & 21.9 & 4 & $75 \cdot 0$ & 89.0 & $96 \cdot 0$ & $118 \cdot 7$ & $92 \cdot 3$ \\
\hline $18 \cdot 5-24.9$ & 104 & 87.5 & $54 \cdot 1$ & 88.7 & 61.8 & $92 \cdot 3$ & 216 & 75.5 & 53.9 & $90 \cdot 0$ & 71.4 & 97.5 \\
\hline$\geq 25 \cdot 0$ & 72 & $75 \cdot 0$ & 64.7 & $111 \cdot 0$ & $86 \cdot 2$ & $120 \cdot 9$ & 97 & $83 \cdot 5$ & $62 \cdot 2$ & 88.9 & 74.5 & 92.5 \\
\hline \multicolumn{13}{|l|}{ Family income (Yuan/person per month) } \\
\hline Less than 2000 & 59 & $83 \cdot 1$ & 79.6 & $131 \cdot 2$ & $95 \cdot 8$ & $138 \cdot 7$ & 121 & 82.6 & 71.6 & 107.5 & $86 \cdot 7$ & $112 \cdot 7$ \\
\hline $2000-4000$ & 55 & $83 \cdot 6$ & $47 \cdot 2$ & $75 \cdot 1$ & $56 \cdot 4$ & $79 \cdot 0$ & 97 & $71 \cdot 1$ & $48 \cdot 0$ & 81.6 & 67.5 & $89 \cdot 8$ \\
\hline $4000-8000$ & 31 & 83.9 & 55.5 & $88 \cdot 1$ & $66 \cdot 1$ & $92 \cdot 6$ & 52 & $80 \cdot 8$ & $41 \cdot 2$ & 61.5 & 51.0 & 64.7 \\
\hline More than 8000 & 10 & $60 \cdot 0$ & 11.6 & $19 \cdot 3$ & $19 \cdot 4$ & $22 \cdot 1$ & 9 & $77 \cdot 8$ & $32 \cdot 6$ & 76.5 & 41.9 & $85 \cdot 8$ \\
\hline \multicolumn{13}{|l|}{ Meals outside the home/week } \\
\hline 0 & 42 & $76 \cdot 2$ & $42 \cdot 4^{*}$ & $69 \cdot 9$ & $55 \cdot 6$ & 75.5 & 97 & $76 \cdot 3$ & $48 \cdot 8$ & $63 \cdot 2$ & 63.9 & $65 \cdot 4$ \\
\hline 1 time & 71 & $83 \cdot 1$ & 49.9 & $82 \cdot 1$ & $60 \cdot 0$ & $86 \cdot 7$ & 118 & $78 \cdot 8$ & 49.5 & $79 \cdot 7$ & $62 \cdot 8$ & 85.0 \\
\hline 2 times & 43 & $86 \cdot 0$ & 91.5 & 138.7 & $106 \cdot 4$ & $144 \cdot 3$ & 72 & 81.9 & $68 \cdot 7$ & 104.7 & 83.8 & $110 \cdot 1$ \\
\hline 3 or more times & 23 & $82 \cdot 6$ & 39.9 & 46.5 & $48 \cdot 3$ & $47 \cdot 0$ & 27 & $70 \cdot 4$ & 61.9 & 78.0 & 87.9 & $79 \cdot 8$ \\
\hline
\end{tabular}


Table 3 Consumption of sugar-sweetened beverages, according to different characteristics and age group, by young children ( $n 419)$ from seven large cities and two villages in China, November 2011-April 2012

\begin{tabular}{|c|c|c|c|c|c|c|c|c|c|c|c|c|}
\hline \multirow[b]{3}{*}{ Characteristic } & \multicolumn{6}{|c|}{$5-5.9$ years } & \multicolumn{6}{|c|}{$6-7$ years } \\
\hline & \multirow[b]{2}{*}{ Sample size } & \multirow[b]{2}{*}{ Consumer (\%) } & \multicolumn{2}{|c|}{ Per capita $(\mathrm{ml} / \mathrm{d})$} & \multicolumn{2}{|c|}{ Per consumer $(\mathrm{ml} / \mathrm{d})$} & \multirow[b]{2}{*}{ Sample size } & \multirow[b]{2}{*}{ Consumer (\%) } & \multicolumn{2}{|c|}{ Per capita $(\mathrm{ml} / \mathrm{d})$} & \multicolumn{2}{|c|}{ Per consumer $(\mathrm{ml} / \mathrm{d}$} \\
\hline & & & Mean & SD & Mean & SD & & & Mean & SD & Mean & SD \\
\hline Total & 299 & 81.9 & 71.6 & 118.9 & 87.4 & $126 \cdot 1$ & 120 & $80 \cdot 0$ & $69 \cdot 3$ & 83.4 & $86 \cdot 6$ & 84.8 \\
\hline \multicolumn{13}{|l|}{ Child's sex } \\
\hline Male & 153 & 83.7 & $80 \cdot 9$ & $140 \cdot 3$ & $96 \cdot 6$ & 148.4 & 55 & $81 \cdot 8$ & $73 \cdot 7$ & $89 \cdot 1$ & $90 \cdot 1$ & $90 \cdot 8$ \\
\hline Female & 146 & $80 \cdot 1$ & 61.9 & $90 \cdot 8$ & $77 \cdot 2$ & 95.5 & 65 & 78.5 & $65 \cdot 6$ & 78.7 & 83.6 & $80 \cdot 0$ \\
\hline \multicolumn{13}{|l|}{ Child's area } \\
\hline Urban & 221 & 81.9 & $59 \cdot 3^{*}$ & $110 \cdot 4$ & $72 \cdot 4^{*}$ & 118.0 & 80 & 81.3 & $48 \cdot 8^{*}$ & $65 \cdot 6$ & $60 \cdot 1^{*}$ & 68.1 \\
\hline Rural & 78 & $82 \cdot 1$ & $106 \cdot 3$ & $135 \cdot 2$ & 129.5 & 138.9 & 40 & $77 \cdot 5$ & $110 \cdot 2$ & 99.5 & $142 \cdot 2$ & $90 \cdot 4$ \\
\hline \multicolumn{13}{|l|}{ Picky eating behaviour } \\
\hline Yes & 143 & $86 \cdot 0$ & $93 \cdot 6^{*}$ & $148 \cdot 3$ & $108 \cdot 9^{*}$ & 154.7 & 52 & 84.6 & $75 \cdot 8$ & 86.0 & $89 \cdot 6$ & $86 \cdot 7$ \\
\hline No & 151 & 77.5 & $52 \cdot 0$ & 79.7 & $67 \cdot 1$ & 84.8 & 66 & $75 \cdot 8$ & 63.0 & 81.5 & 83.2 & $84 \cdot 2$ \\
\hline \multicolumn{13}{|l|}{ Mother's education } \\
\hline Primary school or below & 15 & 93.3 & $84.5^{\star}$ & 91.7 & $90 \cdot 5^{*}$ & $92 \cdot 0$ & 3 & $66 \cdot 7$ & $18 \cdot 0^{*}$ & $24 \cdot 3$ & $27 \cdot 0^{*}$ & $26 \cdot 3$ \\
\hline Middle-school diploma & 88 & 83.0 & 114.9 & $168 \cdot 1$ & 138.5 & 175.6 & 48 & 83.3 & 95.7 & 90.4 & $114 \cdot 8$ & 87.2 \\
\hline High-school diploma & 140 & 83.6 & $53 \cdot 2$ & 88.1 & 63.7 & 92.9 & 44 & 79.5 & $70 \cdot 0$ & $82 \cdot 4$ & 88.1 & 83.4 \\
\hline College degree and above diploma & 50 & $72 \cdot 0$ & $37 \cdot 3$ & 62.5 & 51.8 & 68.5 & 24 & 79.2 & 24.4 & $49 \cdot 2$ & 30.9 & 53.7 \\
\hline \multicolumn{13}{|l|}{ Mother's BMI $\left(\mathrm{kg} / \mathrm{m}^{2}\right)$} \\
\hline$<18.5$ & 33 & $78 \cdot 8$ & 88.6 & 179.6 & 112.5 & $196 \cdot 1$ & 9 & $77 \cdot 8$ & 38.9 & $57 \cdot 2$ & $50 \cdot 0$ & $61 \cdot 0$ \\
\hline $18 \cdot 5-24.9$ & 229 & 82.5 & $68 \cdot 7$ & $107 \cdot 7$ & 83.3 & 113.4 & 92 & 80.4 & $69 \cdot 2$ & $83 . \overline{8}$ & 86.0 & $85 \cdot 3$ \\
\hline$\geq 25 \cdot 0$ & 30 & $80 \cdot 0$ & 81.8 & 131.6 & $102 \cdot 3$ & $140 \cdot 1$ & 13 & 84.6 & $100 \cdot 5$ & $99 \cdot 1$ & 118.8 & $96 \cdot 9$ \\
\hline \multicolumn{13}{|l|}{ Father's education } \\
\hline Primary school or below & 8 & $87 \cdot 5^{\star}$ & $233.6^{*}$ & 346.9 & $267 \cdot 0^{*}$ & $360 \cdot 5$ & 2 & $50 \cdot 0$ & $85 \cdot 7^{\star}$ & $121 \cdot 2$ & - & - \\
\hline Middle-school diploma & 73 & $82 \cdot 2$ & $109 \cdot 0$ & 143.7 & $132 \cdot 6$ & $148 \cdot 3$ & 40 & $90 \cdot 0$ & $121 \cdot 0$ & 101.7 & $134 \cdot 4^{*}$ & 98.4 \\
\hline High-school diploma & 134 & 88.1 & $58 \cdot 8$ & 95.0 & $66 \cdot 8$ & 98.6 & 43 & $76 \cdot 7$ & $55 \cdot 2$ & $60 \cdot 7$ & 71.9 & 59.9 \\
\hline College degree and above diploma & 68 & 69.1 & 34.5 & 49.9 & $50 \cdot 0$ & $53 \cdot 3$ & 31 & $80 \cdot 6$ & 29.7 & 48.9 & $36 \cdot 9$ & $52 \cdot 1$ \\
\hline \multicolumn{13}{|l|}{ Father's BMI $\left(\mathrm{kg} / \mathrm{m}^{2}\right)$} \\
\hline$<18.5$ & 6 & 83.3 & $25 \cdot 7$ & 38.4 & $30 \cdot 8$ & 40.5 & 2 & $100 \cdot 0$ & $76 \cdot 1$ & $74 \cdot 2$ & $76 \cdot 1$ & $74 \cdot 2$ \\
\hline $18.5-24.9$ & 182 & 79.7 & 63.5 & $102 \cdot 5$ & $79 \cdot 7$ & 109.1 & 73 & 80.8 & $64 \cdot 1$ & 76.5 & 79.3 & $77 \cdot 7$ \\
\hline$\geq 25 \cdot 0$ & 101 & $86 \cdot 1$ & $93 \cdot 4$ & $148 \cdot 3$ & $108 \cdot 4$ & $154 \cdot 7$ & 35 & 80.0 & 84.9 & $101 \cdot 8$ & $106 \cdot 1$ & 103.5 \\
\hline \multicolumn{13}{|l|}{ Family income (Yuan/person per month) } \\
\hline Less than 2000 & 113 & $81 \cdot 4^{\star}$ & $93.9^{*}$ & $149 \cdot 3$ & $115 \cdot 3^{*}$ & 158.0 & 53 & $79 \cdot 2$ & 87.4 & $91 \cdot 0$ & $110 \cdot 3$ & $89 \cdot 0$ \\
\hline $2000-4000$ & 78 & 70.5 & $36 \cdot 1$ & 64.1 & $51 \cdot 2$ & $71 \cdot 2$ & 26 & $84 \cdot \overline{6}$ & 59.7 & 85.9 & 70.5 & $89 \cdot 3$ \\
\hline $4000-8000$ & 40 & $95 \cdot 0$ & 67.5 & $104 \cdot 1$ & $71 \cdot 1$ & $105 \cdot 7$ & 17 & 58.8 & $40 \cdot 1$ & $64 \cdot 1$ & $68 \cdot 2$ & 71.9 \\
\hline More than 8000 & 14 & 71.4 & $93 \cdot 0$ & 204.7 & $130 \cdot 2$ & 234.8 & 6 & $100 \cdot 0$ & $42 \cdot 8$ & $19 \cdot 2$ & $42 \cdot 8$ & $19 \cdot 2$ \\
\hline \multicolumn{13}{|l|}{ Meals outside the home/week } \\
\hline 0 & 73 & $75 \cdot 3$ & 45.4 & $62 \cdot 9$ & $60 \cdot 2$ & $66 \cdot 1$ & 32 & $81 \cdot 3$ & $93 \cdot 3$ & 88.0 & 114.8 & 83.9 \\
\hline 1 time & 132 & $86 \cdot 4$ & $80 \cdot 6$ & 133.0 & $93 \cdot 3$ & 139.0 & 47 & 83.0 & 59.4 & $82 \cdot 6$ & 71.6 & 85.9 \\
\hline 2 times & 48 & 83.3 & $78 \cdot 3$ & $116 \cdot 1$ & 94.0 & 121.4 & 23 & 73.9 & $72 \cdot 4$ & 90.0 & 98.0 & $92 \cdot 1$ \\
\hline 3 or more times & 28 & 85.7 & 85.9 & $147 \cdot 1$ & $100 \cdot 2$ & 154.7 & 14 & 92.9 & $62 \cdot 1$ & 63.0 & $66 \cdot 9$ & $62 \cdot 8$ \\
\hline
\end{tabular}

${ }^{*} P<0.05$. 


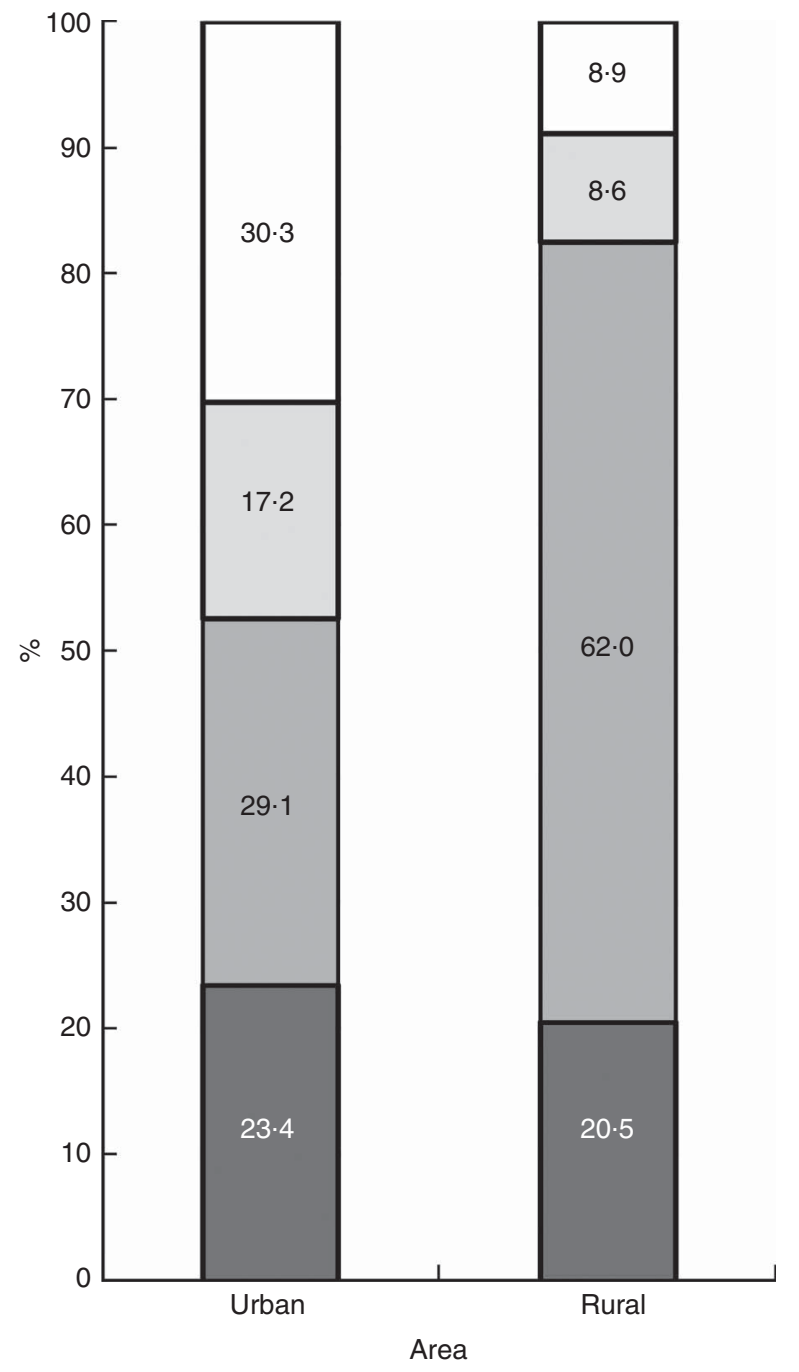

Fig. 1 The types of sugar-sweetened beverages ( $\square$, carbonated beverages; $\square$, tea beverages; $\square$, sports/energy drinks; $\square$, vegetable/fruit-flavoured beverages) consumed, according to area, by young children ( $n$ 946) aged 3-7 years from seven large cities and two villages in China, November 2011-April 2012

confounders (Table 4). To make this finding more comparable with international results, we set $240 \mathrm{ml}$ (about $8 \mathrm{oz}$ ) increments; additionally, taking into account that the results of our own study showed that the mean intake amount of SSB was relatively small, we also set $50 \mathrm{ml}$ increments.

Table 5 shows the results of SSB consumption and the odds of being overweight or obese. Consumers of SSB had a higher risk of being overweight or obese compared with non-consumers, after adjustment for multiple potential confounders.

\section{Discussion}

To the authors' knowledge, the present study is the first to report the SSB consumption among 3-7-year-old children in China. Our study produced three main findings. First, a

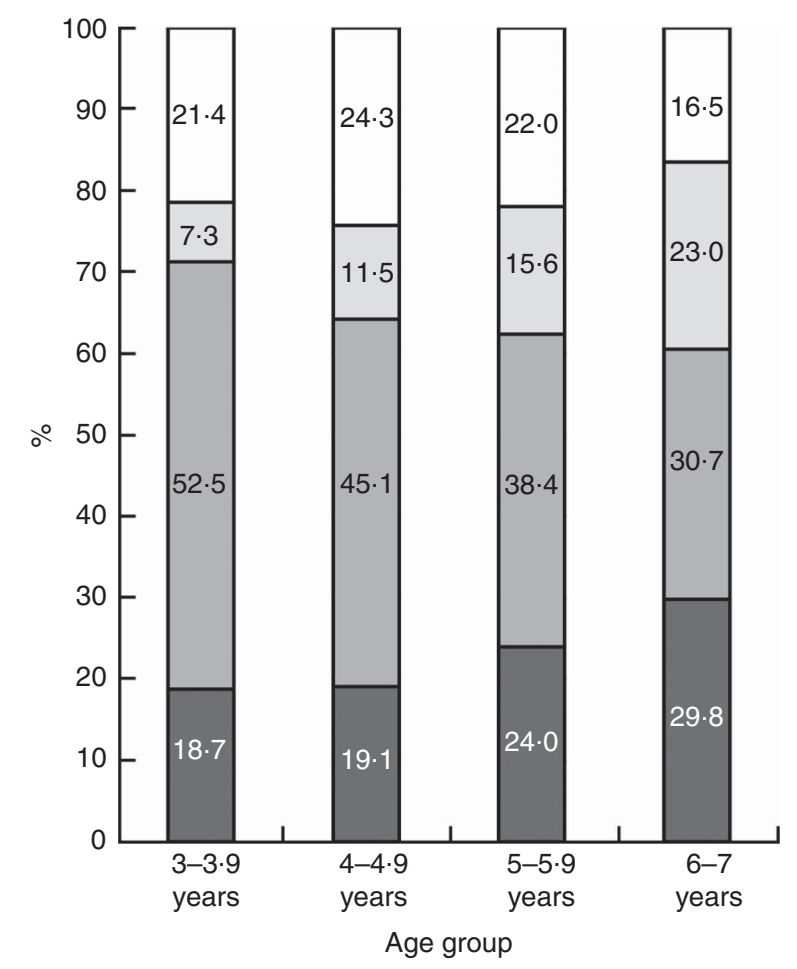

Fig. 2 The types of sugar-sweetened beverages ( $\square$, carbonated beverages; $\square$, tea beverages; $\square$, sports/energy drinks; $\square$, vegetable/fruit-flavoured beverages) consumed, according to age, by young children ( $n$ 946) aged 3-7 years from seven large cities and two villages in China, November 2011-April 2012

particularly high percentage of young children consumed SSB, although the intake amount was relatively small. Second, the children's area (urban or rural) and parents' education levels were the most important factors that impacted the SSB consumption status of children; area affected not only the intake amounts, but also the types of SSB were also significantly different. Third, a positive association was found between SSB intake and weight gain.

Among the whole study population, more than fourfifths $(80.5 \%)$ had consumed SSB during the preceding half year; $35.4 \%$ consumed SSB at least once weekly and $3.4 \%$ at least once daily. These results are similar to those obtained by Huang et al. ${ }^{(15)}$ who, in a study of SSB intake and obesity among urban residents in 2010, showed that about a third (27.2-33.2\%) of teenagers consumed SSB at least once weekly. Similarly, in a report by the Chinese Center for Disease and Prevention, 3.9\% of adults from nine provinces consumed SSB daily in $2009^{(16)}$. However, children from developed countries consume SSB heavily in comparison to Chinese children. For example, $13 \cdot 7-15 \cdot 7 \%$ of children aged $2-5$ years were daily SSB consumers in Canada in 2002 $2^{(26)}$; and in the USA, $9.3 \%$ drank more than one serving (about $240 \mathrm{ml}$ ) daily at 2 years, $13.0 \%$ at 4 years and $11.6 \%$ at 5 years of age ${ }^{(14)}$ On the basis of the 2009-2010 US National Health and Nutrition Examination Survey, energy intake from SSB was $289 \mathrm{~kJ} / \mathrm{d}$, which was equivalent to approximately $180 \mathrm{ml}$ of 


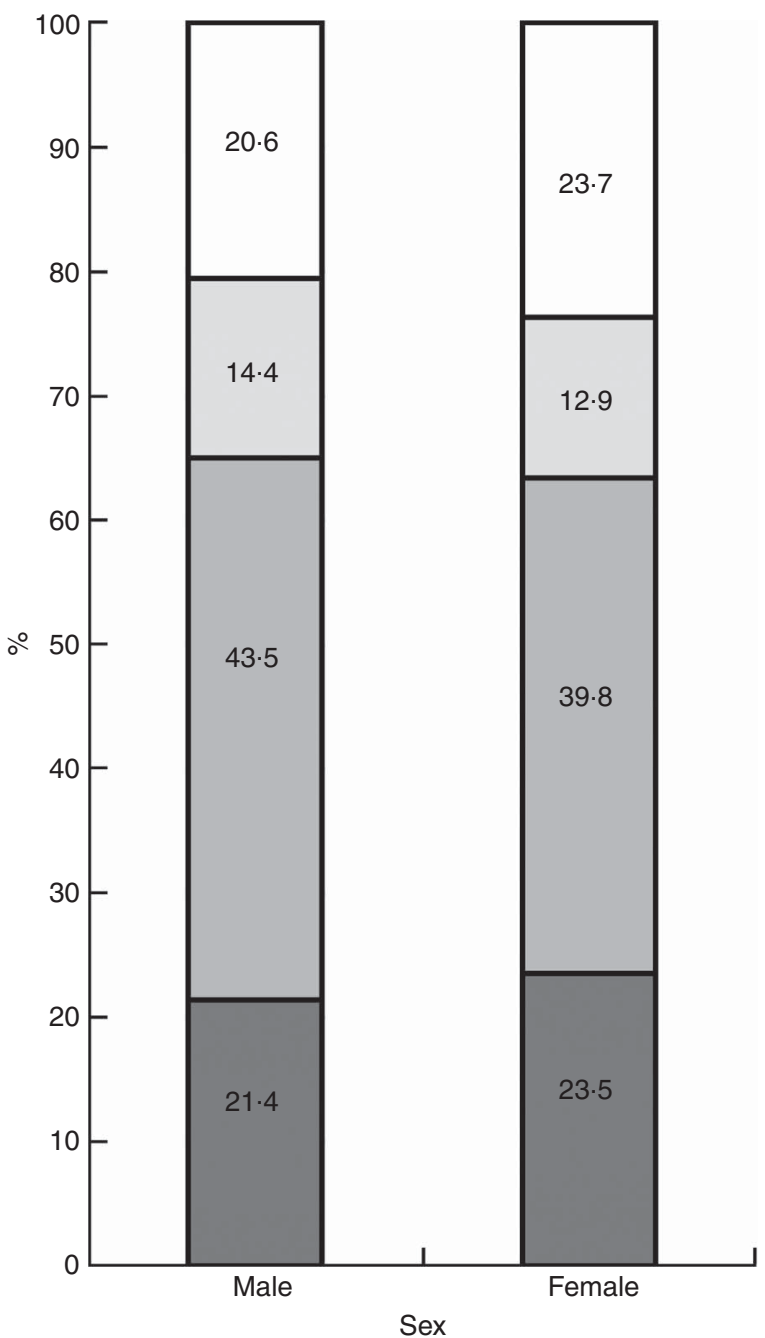

Fig. 3 The types of sugar-sweetened beverages ( $\square$, carbonated beverages; $\square$, tea beverages; $\square$, sports/energy drinks; $\square$, vegetable/fruit-flavoured beverages) consumed, according to sex, by young Chinese children ( $n$ 946) aged 3-7 years from seven large cities and two villages in China, November 2011-April 2012

Table 4 Linear regression model of the association of sugarsweetened beverage (SSB) intake (increasing servings) with BMI-for-age $Z$-score among young children $(n 946)$ aged $3-7$ years from seven large cities and two villages in China, November 2011-April 2012

\begin{tabular}{|c|c|c|c|c|}
\hline & \multicolumn{4}{|c|}{ BMI-for-age Z-score } \\
\hline & Coefficient & SE & Standardised coefficient & $P$ \\
\hline \multicolumn{5}{|c|}{ SSB consumer and non-consumer } \\
\hline Model 0 & 0.312 & 0.134 & 0.076 & 0.20 \\
\hline Model 1 & 0.385 & 0.148 & 0.099 & 0.009 \\
\hline \multicolumn{5}{|c|}{ In $240 \mathrm{ml}$ increments } \\
\hline Model 0 & 0.236 & 0.112 & 0.068 & 0.36 \\
\hline Model 1 & 0.320 & 0.126 & 0.099 & 0.011 \\
\hline \multicolumn{5}{|c|}{ In $50 \mathrm{ml}$ increments } \\
\hline Model 0 & 0.106 & 0.067 & 0.051 & 0.115 \\
\hline Model 1 & 0.210 & 0.077 & 0.107 & 0.007 \\
\hline
\end{tabular}

Model 0, no adjustments; model 1, adjusted for sex, picky eating behaviour, child's area (urban or rural), family income, parents' BMI and education level, and meals outside the home.
Table 5 Logistic regression model of the association of sugarsweetened beverage (SSB) intake with overweight/obesity status among young children ( $n$ 946) aged 3-7 years from seven large cities and two villages in China, November 2011-April 2012

\begin{tabular}{lccc}
\hline SSB & OR & $95 \% \mathrm{Cl}$ & $P$ \\
\hline Model 0 & 1.55 & $0.874,2.739$ & 0.134 \\
Model 1 & 1.58 & $0.889,2.823$ & 0.118 \\
Model 2 & 2.20 & $1.095,4.411$ & 0.027 \\
Model 3 & 2.48 & $1.085,5.649$ & 0.031
\end{tabular}

Model 0 , no adjustments; model 1, adjusted for sex and picky eating behaviour; model 2, adjusted for sex, picky eating behaviour, child's area (urban or rural) and family income); model 3, adjusted for sex, picky eating behaviour, child's area (rural or urban), family income, parents' BMI and education level, and meals outside the home.

soda among children aged $2-5$ years on a given day ${ }^{(27)}$. In our study, the per capita/consumer SSB intake was only 63.1 (sD 100.8)/78.4 (sD 106.9) $\mathrm{ml} / \mathrm{d}$.

Compared with developed countries, China had a relatively lower consumption level; however, this should not lead to complacency, for the following reasons. First, SSB are high in energy and low in nutrition, which is unhealthy for growth and development. A high intake of SSB may lead to an unbalanced diet. Keller et $a l .{ }^{(28)}$ noted that SSB consumption was negatively correlated with both milk $(P<0.01)$ and $\mathrm{Ca}(P<0.01)$ intakes, and these relationships remained after controlling for age, sex and ethnicity $(P<0.01)$. Furthermore, Frary et al. ${ }^{(29)}$ reported that SSB and other sweets decreased the likelihood of meeting the Dietary Reference Intakes for $\mathrm{Ca}$, folate and Fe; only children who were non-consumers of SSB had a mean $\mathrm{Ca}$ intake that met the Adequate Intake recommendation. Furthermore, increased SSB intake is considered to be independently associated with dental caries $^{(30)}$, cardiometabolic markers such as increased C-reactive protein concentrations $(P=0.003)$ and decreased HDL cholesterol concentrations $(P<0.001)$ in young children ${ }^{(31)}$. Other reviews synthesised the evidence in adults and showed effects of SSB on CVD and metabolic syndrome/type 2 diabetes, making it reasonable to speculate that effects on intermediate outcomes may lead to insulin resistance, diabetes and dyslipidaemia in children ${ }^{(32)}$. Second, it is important to note that young children are going through a special period of life, wherein food preferences and eating patterns developed in early childhood appear to continue into adolescence and adulthood $^{(33)}$.

Higher SSB intake in children in the present study was associated with various characteristics such as sex, child's area (urban or rural), picky eating behaviour, parents' education and BMI, and family income. Among all the factors, area and parents' education level were the most important. The amount of SSB intake of boys was higher than that of girls (72.6 (sD 118.5) v. 52.8 (sD 75.9) $\mathrm{ml} / \mathrm{d}$; $P<0.05)$, which is comparable with previous results ${ }^{(28)}$. Among the different age groups, a significant difference between boys and girls existed only in the 3-3.9 year age 
group. Children with a picky eating habit had a much higher intake of SSB; a possible reason for this may be that parents were inclined to gratify the desires of their children, however further studies are needed. Similarly to other reports, our study also indicated that children whose parent(s) had a relatively lower education level and/or with a low family income consumed much more $\mathrm{SSB}^{(17,34)}$. These results may imply that low-income individuals may access SSB more easily than nutrient-dense beverages, such as milk. A different consumption status in children of parents with a lower education level may indicate the importance of the household environment in guiding healthy beverage choices. This speculation is strengthened by the significant differences in SSB consumption between urban and rural children. Contrary to our expectations, except for the 3-3.9 year old group, we did not find differences among children according to the amount of meals they ate outside the home per week. We speculate that children whose parents had a lower education level and/or with a low family income may have fewer chances to eat out, while the other children may eat out more and access SSB more easily; however, further studies are needed to investigate this further.

The types of SSB consumed by Chinese young children are different from those among American children; fruit drinks (non-100\% fruit juices) were consumed the most in America $^{(34)}$, while tea beverages $(42 \cdot 1 \%)$ were the most heavily consumed SSB among Chinese children. The consumption patterns of SSB between the sexes were similar; however, there were significant differences between ages and areas. From 3 to 7 years, there was a decreasing trend of tea beverage consumption along with an increasing trend of carbonated and sports/energy beverage consumption. It is known that carbonated beverages are the most heavily consumed SSB among adolescents and adults in America ${ }^{(34)}$ and it may be reasonable to speculate that with ageing, carbonated beverages may become the major SSB in China; we need to take serious note of this.

Among urban children, the most consumed SSB were vegetable/fruit-flavoured drinks (30.3\%), similar to American children $^{(34)}$, followed by tea beverages (29.1\%), carbonated beverages (23.4\%) and sports/energy drinks (17.2\%). However, among rural children, the most heavily consumed SSB were tea beverages, which accounted for up to $62.0 \%$ of all the SSB consumed, followed by carbonated beverages (20.5\%), vegetable/fruit-flavoured beverages (8.9\%) and sports/energy drinks (8.6\%). Previously, we found that about $70 \%$ of urban pupils consumed liquid dairy products frequently (more than four times per week), which was higher than rural children $(22.2 \%, P<0.05)$; and urban children consumed an average of $206 \mathrm{ml}$ liquid dairy/d compared with $44 \mathrm{ml}$ liquid dairy consumed by rural children daily $(P<0 \cdot 05)^{(35)}$. Disparities in urban-rural health and nutrition of children have been declining significantly from 1989 to $2006^{(36)}$; however, from our study, we still can see great differences between urban and rural areas regarding drink consumption. About 15 years ago, among urban children and adolescents, 86.9, $58 \cdot 0,37 \cdot 0$ and $16 \cdot 2 \%$ drank plain water, milk, carbonated beverages and fruit-flavoured juices during a day ${ }^{(37)}$. In $2008,57 \cdot 6,52 \cdot 5$ and $50.6 \%$ of urban children aged 8-12 years were regular consumers of tea beverages, carbonated beverages and vegetable/fruit-flavoured juices, respectively $^{(38)}$. Drink consumption patterns have changed along with development and urbanisation, and also changes in the drinks industry. Vegetable/fruit-flavoured drinks and tea beverages have become the leading SSB in China nowadays; advertisements for them can be seen everywhere.

Whether SSB intake is associated with weight gain still remains controversial ${ }^{(7)}$, but our study manifested a positive result: with the increasing intake of SSB, BMI-for-age $Z$-scores increased and, compared with non-consumers, SSB consumers had a higher risk of being overweight or obese. Because of the high added-sugar content, low satiety and an incomplete compensatory reduction in energy intake at subsequent meals after the intake of liquid energy, SSB may lead to weight gain ${ }^{(12)}$. In addition, fructose from any sugar, or high-fructose corn syrup, has been shown to promote development of visceral adiposity and ectopic fat deposition ${ }^{(11)}$. However, until now, it is still difficult to draw a definitive conclusion as to whether SSB are significantly implicated in weight gain. Further comprehensive studies are necessary.

In spite of controversial results regarding the association between SSB intake and weight gain among young children, efforts should be made to reduce the consumption of SSB among children. By 2004, many states and school districts had adopted policies that limited access to SSB in the $\mathrm{USA}^{(39)}$; and in March 2014, the WHO released draft guidelines with recommendations on limiting sugar intake (through food and/or beverages) to reduce public health problems such as overweight, obesity and dental caries ${ }^{(40)}$. Studies also investigated effective measures to reduce SSB consumption. For instance, it is effective to substitute water, milk or other more healthy drinks for $\mathrm{SSB}^{(41,42)}$. Compared with schools with no vending machines, daily servings increased by 0.21 for one to three purchases per week $(P=0.0057)$, while 0.71 with four or more purchases $(P=0.0001)$ in schools with vending machines ${ }^{(43)}$; hence, schools with less SSB exposure have fewer students consuming $\mathrm{SSB}^{(44)}$. Furthermore, a tax on SSB would have strong positive effects on reducing SSB consumption ${ }^{(43)}$. Children spend most of their time in school and efforts targeted at building a healthier school environment in China are required. The following recommendations may be helpful:

1. Children should be encouraged to replace SSB with more healthy beverages, such as water. Parents should be encouraged to provide water for their children. At school, teachers and recruited peer educators may help to decrease SSB intake. 
2. Policies restricting the sale of $S S B$ at and around school should be supported.

3. Advertising of SSB should be limited, especially on children's television channels.

4. A healthy attitude towards drinks should be promoted by the Ministry of Education and health education institutes.

Our study has comprehensively reported the SSB consumption status of young children aged 3-7 years in China and found a positive association between SSB intake and weight gain; however, several limitations should be considered. First, China covers a vast geographic area with various customs. The rural sample from two areas of Hebei Province was not a nationally representative sample. Second, important potential confounders that may have affected the results, such as physical status and television viewing time, were not available in our study.

\section{Conclusions}

In summary, the SSB consumption status of young children in China is of concern. There is great disparity of SSB intake between urban and rural areas; targeted health education is needed urgently to help children form healthy habits when consuming drinks. Efforts from family, school, government and society should be encouraged to reduce SSB consumption in children to prevent potential and actual health risks.

\section{Acknowledgements}

Acknowledgements: The authors wish to thank all participants in the study. The study was carried out in cooperation with the School of Public Health at Suzhou University, Guangzhou University of Chinese Medicine, Zhengzhou University, and several other universities in China. Financial support: This research project was part of a larger child nutrition study, sponsored by Mengniu Dairy Co. Ltd (Inner Mongolia, China). This research project was a collaborative work between Peking University and Mengniu Dairy Co. Ltd (Inner Mongolia, China). The views and opinions expressed in this manuscript are those of the authors and do not necessarily reflect the opinions and recommendations of Mengniu Dairy Co. Ltd (Inner Mongolia, China). This study was funded by Key Projects of Beijing Science \& Technology (grant number Z1411000048140); the Natural Science Foundation of Beijing (grant number Z140001); and the Natural Science Foundation of China (grant number 81573219). Conflicts of interest: The investigation was supported by Mengniu Dairy Co. Ltd (Inner Mongolia, China) and the authors declare that they have no competing interests. Authorship: Y.M.Z., Y.B. and Y.D.Z. conceived and led the study, A.Z., Y.C. and W.Z.Z. coordinated data collection. P.Y. conducted the analyses and was the primary writer of the manuscript. All authors contributed to the interpretation of the data and the writing of the manuscript. Ethics of buman subject participation: The study protocol was reviewed and approved by ethical review committees in Peking University Health Science Center (number IRB00001052-11042) and all participants gave their written and informed consent signed by their legal guardians.

\section{References}

1. World Health Organization (2014) Global Nutrition Targets 2025: Childhood Overweight Policy Brief (WHO/NMH/ NHD/14.6). Geneva: WHO.

2. Yu Z, Han S, Chu J et al. (2012) Trends in overweight and obesity among children and adolescents in China from 1981 to 2010: a meta-analysis. PLoS One 7, e51949.

3. Nader PR, O'Brien M, Houts R et al. (2006) Identifying risk for obesity in early childhood. Pediatrics 118, e594-e601.

4. Franks PW, Hanson RL, Knowler WC et al. (2010) Childhood obesity, other cardiovascular risk factors, and premature death. N Engl J Med 362, 485-493.

5. Duffey KJ \& Popkin BM (2007) Shifts in patterns and consumption of beverages between 1965 and 2002. Obesity (Silver Spring) 15, 2739-2747.

6. Libuda L \& Kersting M (2009) Soft drinks and body weight development in childhood: is there a relationship? Curr Opin Clin Nutr Metab Care 12, 596-600.

7. Trumbo PR \& Rivers CR (2014) Systematic review of the evidence for an association between sugar-sweetened beverage consumption and risk of obesity. Nutr Rev $\mathbf{7 2}$, 566-574.

8. Massougbodji J, Le Bodo Y, Fratu R et al. (2014) Reviews examining sugar-sweetened beverages and body weight: correlates of their quality and conclusions. Am J Clin Nutr 99, 1096-1104.

9. Gibson S (2008) Sugar-sweetened soft drinks and obesity: a systematic review of the evidence from observational studies and interventions. Nutr Res Rev 21, 134-147.

10. Forshee RA, Anderson PA \& Storey ML (2008) Sugarsweetened beverages and body mass index in children and adolescents: a meta-analysis. Am J Clin Nutr 87, 1662-1671.

11. Malik VS, Pan A, Willett WC et al. (2013) Sugar-sweetened beverages and weight gain in children and adults: a systematic review and meta-analysis. Am J Clin Nutr 98, 1084-1102.

12. Malik VS, Schulze MB \& Hu FB (2006) Intake of sugarsweetened beverages and weight gain: a systematic review. Am J Clin Nutr 84, 274-288.

13. Ludwig DS, Peterson KE \& Gortmaker SL (2001) Relation between consumption of sugar-sweetened drinks and childhood obesity: a prospective, observational analysis. Lancet 357, 505-508.

14. DeBoer MD, Scharf RJ \& Demmer RT (2013) Sugarsweetened beverages and weight gain in 2- to 5-year-old children. Pediatrics 132, 413-420.

15. Huang Q, Shao J, Xu L et al. (2013) The relationship between the consumption of sugar-sweetened beverages and obesity in city dwellers. Acta Univ Med Anbui 48, 30-33.

16. Li D, Yu D \& Zhao L (2014) Trend of sugar-sweetened beverage consumption and intake of added sugar in China nine provinces among adults. J Hyg Res 43, $70-72$.

17. Shang XW, Liu AL, Zhang Q et al. (2012) Report on childhood obesity in China (9): sugar-sweetened beverages consumption and obesity. Biomed Environ Sci 25, 125-132. 
18. Gordon-Larsen P, Wang H \& Popkin BM (2014) Overweight dynamics in Chinese children and adults. Obes Rev 15, Suppl. 1, 37-48.

19. Xue Y, Lee E, Ning K et al. (2015) Prevalence of picky eating behaviour in Chinese school-age children and associations with anthropometric parameters and intelligence quotient. A cross-sectional study. Appetite 91, 248-255.

20. Kosova EC, Auinger P \& Bremer AA (2013) The relationships between sugar-sweetened beverage intake and cardiometabolic markers in young children. J Acad Nutr Diet 113, 219-227.

21. Nickelson J, Lawrence JC, Parton JM et al. (2014) What proportion of preschool-aged children consume sweetened beverages? J Sch Health 84, 185-194.

22. World Health Organization (2007) WHO AnthroPlus Software: A Software for the Global Application of the WHO Reference 2007 for 5-19 Years to Monitor the Growth of School-Age Children and Adolescents. Geneva: WHO.

23. World Health Organization (2011) WHO Anthro (version 3.2.2, January 2011) and macros. http://www.who.int/ childgrowth/software/en/ (accessed May 2015).

24. World Health Organization (2008) Training Course on Child Growth Assessment. Geneva: World Health Organization.

25. World Health Organization (2007) Growth reference 5-19 years. http://www.who.int/growthref/who2007_bmi_for_ age/en/ (accessed December 2015).

26. Dubois L, Farmer A, Girard M et al. (2007) Regular sugarsweetened beverage consumption between meals increases risk of overweight among preschool-aged children. $J$ Am Diet Assoc 107, 924-934.

27. Kit BK, Fakhouri TH, Park S et al. (2013) Trends in sugar-sweetened beverage consumption among youth and adults in the United States: 1999-2010. Am J Clin Nutr 98, 180-188.

28. Keller KL, Kirzner J, Pietrobelli A et al. (2009) Increased sweetened beverage intake is associated with reduced milk and calcium intake in 3- to 7-year-old children at multi-item laboratory lunches. J Am Diet Assoc 109, 497-501.

29. Frary CD, Johnson RK \& Wang MQ (2004) Children and adolescents' choices of foods and beverages high in added sugars are associated with intakes of key nutrients and food groups. J Adolesc Health 34, 56-63.

30. Armfield JM, Spencer AJ, Roberts-Thomson KF et al. (2013) Water fluoridation and the association of sugar-sweetened beverage consumption and dental caries in Australian children. Am J Public Health 103, 494-500.
31. Kosova EC, Auinger P \& Bremer AA (2013) The relationships between sugar-sweetened beverage intake and cardiometabolic markers in young children. J Acad Nutr Diet 113, 219-227.

32. Stevens A, Hamel C, Singh K et al. (2014) Do sugarsweetened beverages cause adverse health outcomes in children? A systematic review protocol. Syst Rev 3, 96.

33. Birch LL (1999) Development of food preferences. Annu Rev Nutr 19, 41-62.

34. Han E \& Powell LM (2013) Consumption patterns of sugarsweetened beverages in the United States. J Acad Nutr Diet 113, 43-53.

35. You L, Yang T, Bai Y et al. (2014) Consumption pattern of liquid dairy products and soft beverages among pupils from nine areas in China. Chin J Sch Health 35, $1772-1775$.

36. Liu H, Fang $H \&$ Zhao Z (2013) Urban-rural disparities of child health and nutritional status in China from 1989 to 2006. Econ Hum Biol 11, 294-309.

37. Ma W, Ma G, Hu X et al. (2001) The beverage consumption practice of Chinese children in four urban areas. Chin J Sch Health 22, 102-104.

38. Liu A, Duan Y, Hu X et al. (2011) Change in snacking behaviors of children in four cities of China over 10 years. Chin J Sch Health 32, 1415-1417.

39. Mello MM, Pomeranz J \& Moran P (2008) The interplay of public health law and industry self-regulation: the case of sugar-sweetened beverage sales in schools. Am J Public Health 98, 595-604.

40. World Health Organization (2014) WHO Draft Guideline: Sugars Intake for Adults and Children. http://www.who.int/ nutrition/sugars_public_consultation/en/ (accessed May 2015).

41. de Ruyter JC, Olthof MR, Seidell J et al. (2012) A trial of sugar-free or sugar-sweetened beverages and body weight in children. $N$ Engl J Med 367, 1397-1406.

42. van de Gaar VM, Jansen W, van Grieken A et al. (2014) Effects of an intervention aimed at reducing the intake of sugar-sweetened beverages in primary school children: a controlled trial. Int J Behav Nutr Phys Act 11, 98.

43. Wiecha JL, Finkelstein D, Troped PJ et al. (2006) School vending machine use and fast-food restaurant use are associated with sugar-sweetened beverage intake in youth. J Am Diet Assoc 106, 1624-1630.

44. Johnson DB, Bruemmer B, Lund AE et al. (2009) Impact of school district sugar-sweetened beverage policies on student beverage exposure and consumption in middle schools. $J$ Adolesc Health 45, 3 Suppl., S30-S37. 